\title{
Effects of horizontal vibration on hopper flows of granular materials
}

\author{
M. L. Hunt, R. C. Weathers, A. T. Lee, and C. E. Brennen \\ Division of Engineering and Applied Science, California Institute of Technology, Pasadena, California 91125 \\ C. R. Wassgren \\ Department of Mechanical Engineering, Purdue University, West Lafayette, Indiana 47907-1288
}

(Received 17 November 1997; accepted 2 October 1998)

\begin{abstract}
The current experiments investigate the discharge of glass spheres in a planar wedge-shaped hopper $\left(45^{\circ}\right.$ sidewalls) that is vibrated horizontally. When the hopper is discharged without vibration, the discharge occurs as a funnel flow, with the material exiting the central region of the hopper and stagnant material along the sides. With horizontal vibration, the discharge rate increases with the velocity of vibration as compared with the discharge rate without vibration. For a certain range of acceleration parameters $(20-35 \mathrm{~Hz}$ and accelerations greater than about $1 \mathrm{~g})$, the discharge of the material occurs in an inverted-funnel pattern, with the material along the sides exiting first, followed by the material in the core; the free surface shows a peak at the center of the hopper with the free surface particles avalanching from the center toward the sides. During the deceleration phase of a vibration cycle, particles all along the trailing or low-pressure wall separate from the surface and fall under gravity for a short period before reconnecting the hopper. For lower frequencies $(5$ and 10 $\mathrm{Hz}$ ), the free surface remains horizontal and the material appears to discharge uniformly from the hopper. (C) 1999 American Institute of Physics. [S1070-6631(99)02201-1]
\end{abstract}

\section{INTRODUCTION}

The discharge of granular material from a hopper or bin is a common occurrence in many different types of industries. $^{1,2}$ The geometry and surface conditions of the hopper and the characteristics of the stored material dictate the flow patterns and discharge rates. In general, hoppers are classified in two different types: mass flow and funnel flow. In a mass-flow hopper, all of the material moves as the hopper is discharged; in a funnel-flow design, the movement of the material is confined to the vertical region in the center of the hopper. Hence, for mass-flow hoppers, the material that enters the hopper first is discharged first; in a funnel flow hopper, the material that enters first is discharged last. The inclined walls of a mass-flow hopper must be steep, with the necessary angle dependent on the type of material involved. To date, the problem of predicting the necessary angle for mass flow and the resulting discharge rate relies on empirical data. ${ }^{3-5}$

A simple analysis of a discharging hopper suggests the functional dependence of the discharge rate on the hydraulic diameter, $D_{h} \cdot{ }^{1,2}$ From these studies the discharge rate, $W$, for a particular planar hopper should vary as follows:

$$
W \propto \rho_{b} A\left(g D_{h}\right)^{1 / 2},
$$

where $\rho_{b}$ is the bulk density of the material, $g$ is the gravitational constant, $A$ is the exit area of the hopper, $D_{h}$ is the hydraulic diameter that is calculated from the width, $b$, and depth, $l$, of the hopper. To predict the mass flow rate for different sizes of granular materials, empirical studies suggest that the hydraulic diameter appearing in Eq. (1) be replaced with the reduced hydraulic diameter, $D_{h}^{\prime}$ based on a reduced width, $b^{\prime}$, and depth, $l^{\prime} .{ }^{1-3}$ The reduced dimensions are used because the flow is radial at the exit of a hopper, leaving an empty space around the edge of the orifice that cannot be occupied by a solid particle of diameter, $d$. Generally, the corrected lengths are defined as $b^{\prime}=b-k d$ and $l^{\prime}=l-k d$, with $k$ ranging from 1.3 to 2.9 depending on the characteristics of the materials. ${ }^{3}$ With these modifications, the discharge rate is expressed as

$$
W=C \rho_{b} A^{\prime}\left(g D_{h}^{\prime}\right),
$$

where $A^{\prime}$ is the corrected area based on $b^{\prime}$ and $l^{\prime}$. The constant $C$ is of order 1 and depends on the type of material, the discharge angle, the hopper geometry and the surface conditions.

To increase the mobility of material within a hopper, a "live-wall" design is often used in which an out-of-balance motor is attached to the side of the hopper. By vibrating the wall in both the vertical and the horizontal directions, these motors can maintain a continuous movement of the material when placed in an appropriate position. Alternatively, industries might also use "bin activators" to vibrate locally a section of the hopper; these devices may rely on either purely horizontal, or purely vertical vibration.

The present study examines the effect of horizontal vibration on the discharge of material from a planar hopper in which the entire hopper is vibrated on a shaker table. The objective is to understand the flow patterns induced by the vibration to aid in better designs of vibrating hoppers. There have been earlier studies on vertically vibrating hoppers, ${ }^{6-8}$ indicating that vertical vibration could either increase or decrease the discharge rate depending on the vibration velocity and acceleration. Two recent studies ${ }^{9,10}$ have considered horizontal vibration in a contained layer of granular material. Ristow et al. ${ }^{9}$ showed a transition from solid-like to fluidlike behavior corresponded to an acceleration of $1 \mathrm{~g}$; Liffman 


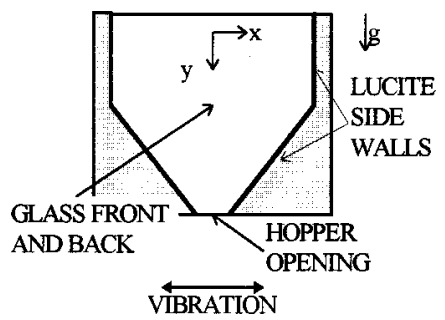

FIG. 1. Diagram of horizontal vibrating hopper.

et al. ${ }^{10}$ examined convective effects due to gap formation between the walls and the bed.

\section{EXPERIMENTAL FACILITY AND METHOD}

The planar hopper used in the experiments is shown in Fig. 1. The vertical front and backsurfaces are double layered, with the inner surface made from 3-mm-thick tempered glass and the outer surface of $12.7 \mathrm{~mm}$ Lucite $^{\mathrm{TM}}$. The purpose of the inner glass plates is to minimize electrostatic charge and to maximize the flow slippage along the surface. The Lucite outer walls provide structural integrity for the hopper. The $45^{\circ}$ sidewalls are smooth-milled Lucite surfaces. The depth of the hopper is $1.27 \mathrm{~cm}$ and the hopper opening is $1.75 \mathrm{~cm}$. The distance between the sidewalls is $29.75 \mathrm{~cm}$, the overall height is $33 \mathrm{~cm}$, and the height of the bin above the $45^{\circ}$ sidewalls is $19 \mathrm{~cm}$. A thin metal plate blocks the hopper opening and is withdrawn to initiate the discharge experiments.

The hopper is mounted on a thick aluminum plate that is attached to the shaker table. The table provides sinusoidal, horizontal vibrations with variable frequency and acceleration. A metal collection bucket (grounded to minimize static electricity) is mounted to the aluminum plate below the hopper. The materials used are spherical glass beads of 1.1 or 2.0 $\mathrm{mm}$ diam. For some flow visualization experiments, the beads were dyed with a water-based paint. The beads and the hopper were periodically washed to minimize the effects of dirt and static electricity.

The $x$ position can be represented by $x=a \sin \omega t$, where $a$ is the amplitude of the vibration, and $\omega$ is the angular frequency (the frequency, $f=\omega / 2 \pi$ is also used). The vibration of the plate is described by its maximum velocity, $a \omega$, or acceleration, $a \omega^{2}$. In addition, the position of the plate may be defined in terms of the angle, $\theta$, where $\theta=$ $(-1)^{n}(\omega t-n \pi)$ with $n=0,1,2, \ldots$ and $-\pi / 2<\theta<\pi / 2$. The two governing nondimensional parameters are the acceleration amplitude, $\Gamma=a \omega^{2} / g$, and the vibration amplitude, $\Omega=a \omega / V$, where $V$ is a characteristic velocity of discharge, which is taken as $V=\left(g D_{h}^{\prime}\right)^{1 / 2}$. The inclination angle of the hopper with respect to the horizontal is denoted by $\alpha$.

In the first set of experiments, the discharge rate of the material was measured for both 1.1 and $2.0 \mathrm{~mm}$ beads. The vibrational frequency ranged from 5 to $35 \mathrm{~Hz}$, and acceleration from 0 to a maximum of $3.0 \mathrm{~g}$ at the highest frequency. At lower frequencies, the maximum acceleration was limited because of the capabilities of the shaker.

For each experiment, $1.2 \mathrm{~kg}$ of material was placed in the hopper. The flow was initiated by manually removing the

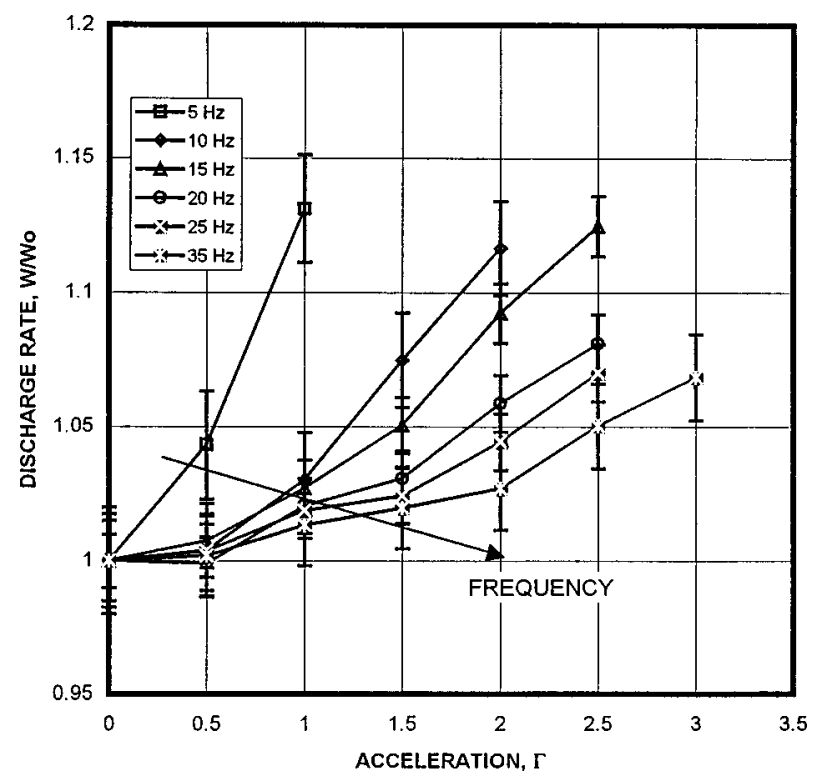

FIG. 2. Discharge rate divided by the discharge rate without vibration for $1.1 \mathrm{~mm}$ glass beads as a function of acceleration and frequency.

hopper plug. The discharge rate was timed, and the rate was compared to the discharge rate without vibration measured in an experiment on the same day. Day to day variations occurred due to temperature, humidity, and static charge. The discharge rates were averaged over six measurements, and the subsequent figures include an indication of the deviation in the measurements. The average discharge time without vibration was $9.56 \mathrm{~s}$ for $1.1 \mathrm{~mm}$ spheres and $10.7 \mathrm{~s}$ for 2.0 $\mathrm{mm}$ spheres. The discharging material was visualized using either a standard 30 fps charge-coupled device (CCD) camera or a high-speed (up to $500 \mathrm{fps}$ ) digital camera.

\section{DISCHARGE RATES}

Figures 2 and 3 present the discharge rate, $W$, divided by the discharge rate without vibration, $W_{0}$, for both the 1.1 and $2.0 \mathrm{~mm}$ glass beads as a function of the nondimensional acceleration amplitude, $\Gamma$. Clearly, the discharge rate increases with vibrational acceleration. However, at the higher frequencies, the increase in discharge rate does not occur until the acceleration amplitude is greater than approximately $\Gamma \approx 1$. The discharge rate also depends on the frequency of vibration, with the lowest frequencies resulting in the highest discharge rates for a fixed acceleration level.

Figure 4 presents the same discharge results in terms of the nondimensional vibrational velocity, $\Omega$. The results fall closer together, suggesting that the discharge rate is strongly dependent on the vibrational velocity. Thus, for a fixed $\Gamma$, the discharge rate increases with vibrational velocity. Figure 4 also shows that below $\Omega \approx 0.2$ there is negligible effect of the vibration on the discharge rate. In addition, the difference between the results for the 1 and the $2 \mathrm{~mm}$ spheres is small. A nominal value of $k=1.4$ is used to define the characteristic velocity of discharge. ${ }^{3}$ 


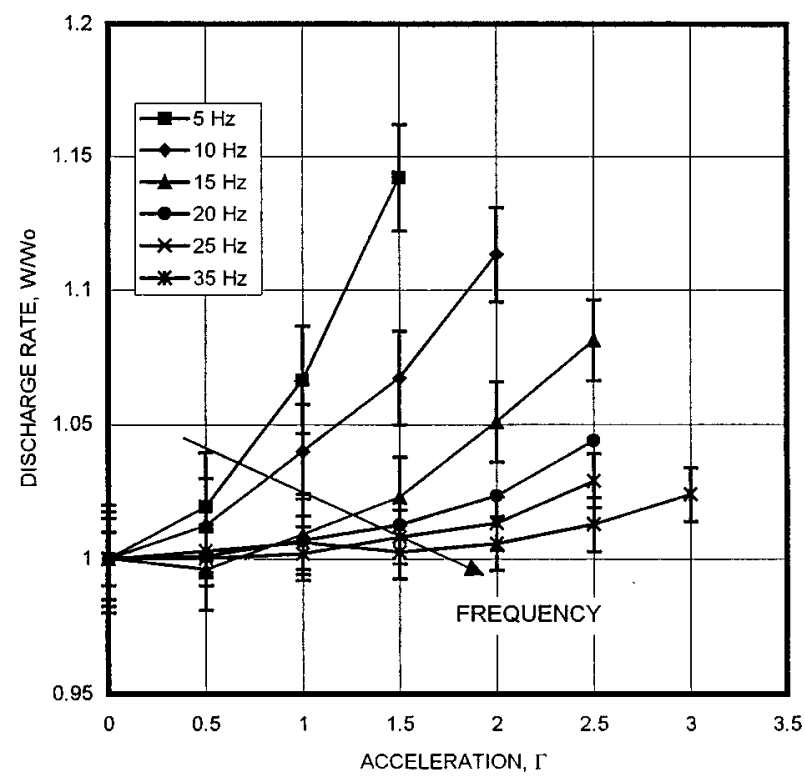

FIG. 3. Discharge rate divided by the discharge rate without vibration for $2.0 \mathrm{~mm}$ glass beads as a function of acceleration and frequency.

\section{FEATURES OF THE DISCHARGING FLOW}

The flow was visualized by loading the hopper with alternate horizontal layers of colored and noncolored particles. Figures 5(a)-5(d) show digitized images without vibration. Clearly, the material is first discharged from the central region of the hopper. The material then avalanches down the sloping upper surfaces so that the material adjacent to the walls discharges last. The upper surface is inclined at approximately $60^{\circ}$ with respect to the vertical.

Figures $6(a)-6(d)$ show the flow patterns for a vibration of $\Gamma=2.0$ at $20 \mathrm{~Hz}$. In Fig. 6(a), the material is not flowing and the only visible movement of the material occurs in the upper corners of the hopper. The material moves down along

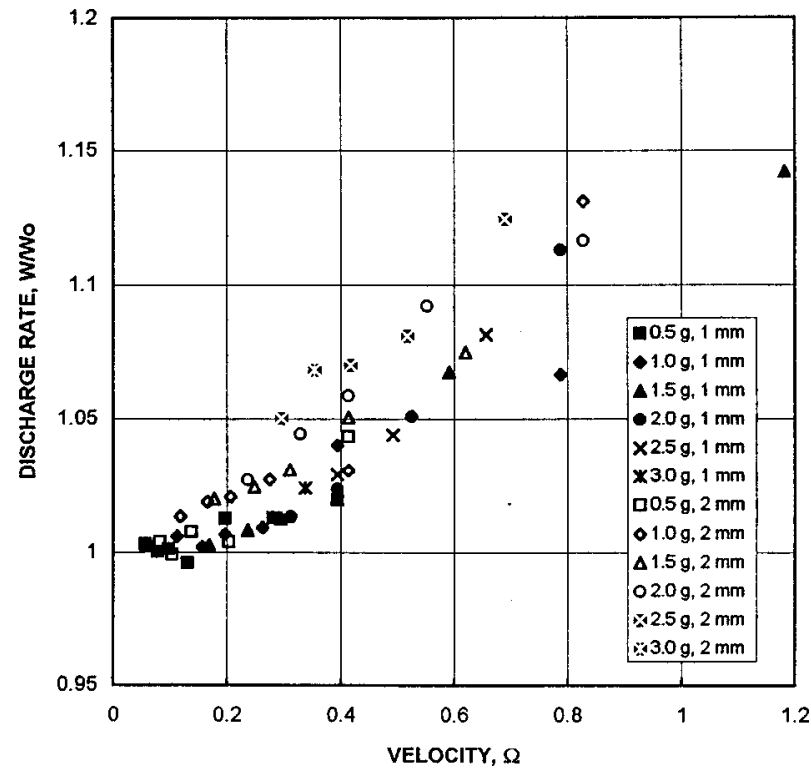

FIG. 4. Discharge rate for 1.1 and $2.0 \mathrm{~mm}$ glass beads as a function of nondimensional velocity amplitude, $\Omega=(a \omega) /\left(g D_{h}^{\prime}\right)^{1 / 2}$.
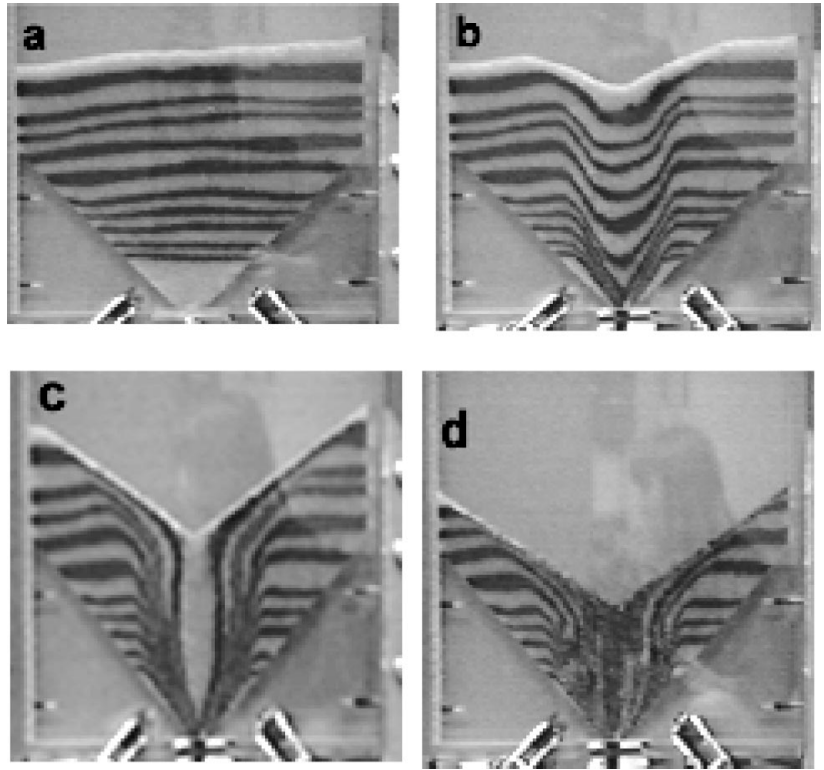

FIG. 5. Digitized images of the hopper discharging without vibration showing the funnel-flow pattern; (a) the initial layering of the hopper; (b)-(d) at different points during the discharge.

the vertical walls and then circulates up through the bed of material. The result of this motion is the triangular mixed region observed in Fig. 6, which does not increase in size after the initial transient period. Under close inspection, it was observed that a momentary gap opens between the sides of the hopper and the material during one part of the oscillation cycle; this gap was also observed by Liffman et al. ${ }^{10}$ After the hopper reverses direction, the material and the hopper wall reconnect. The gap extends to the corner between the vertical and sloped walls.
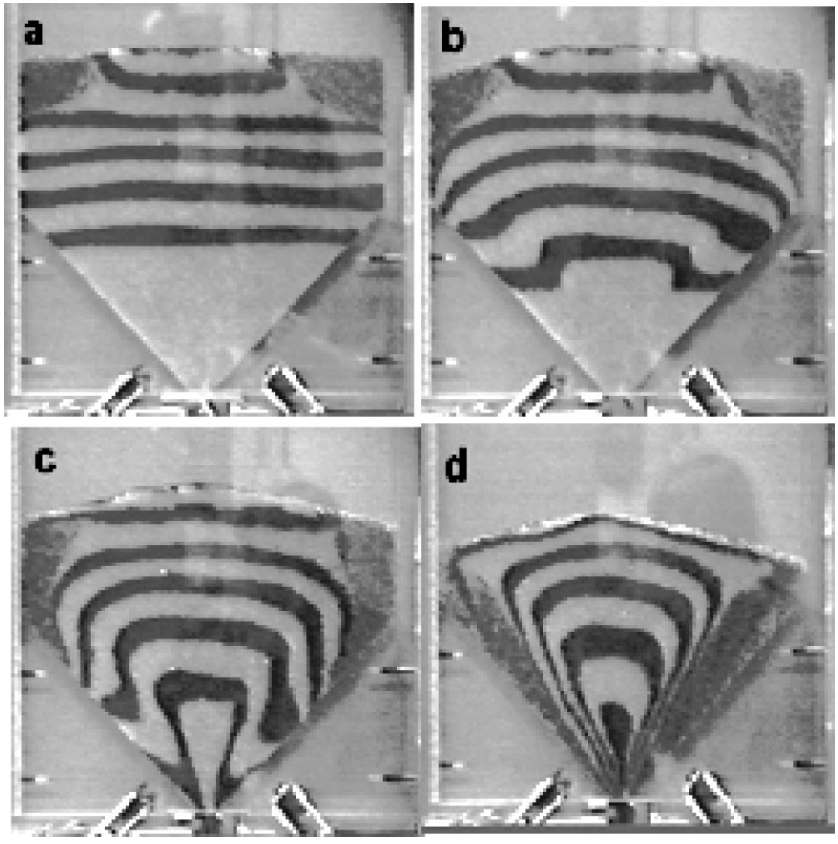

FIG. 6. Digitized images of the hopper discharging with vibration showing the inverted-funnel flow pattern; (a) with the hopper closed; (b)-(d) at different points during the discharge. 


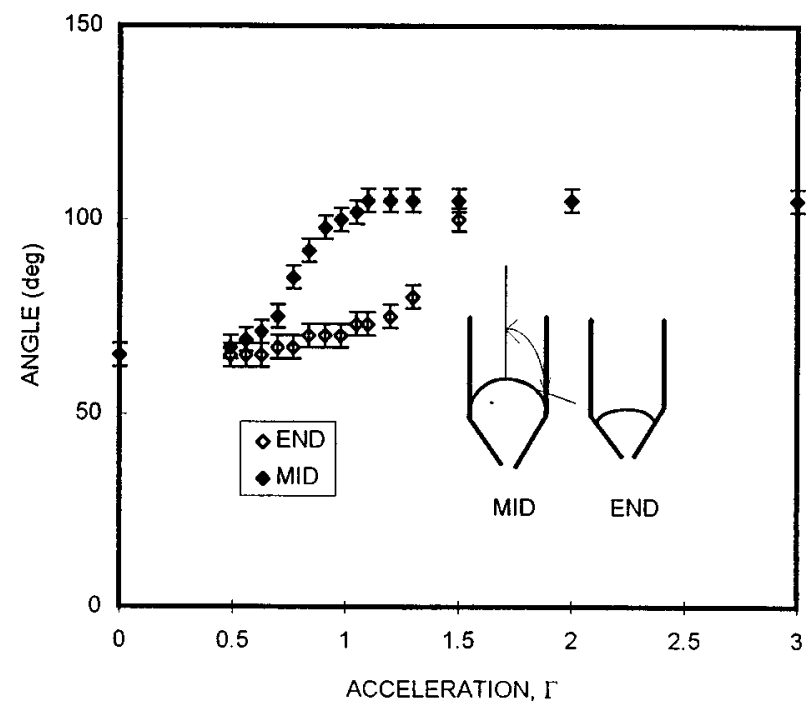

FIG. 7. Inclination of the free surface for vibration at $35 \mathrm{~Hz}$ and a range of accelerations. The filled symbols are measurements taken midway through the discharge, and the open symbols for data during the last $20 \%$ of discharge.

When the hopper is discharged as seen in Figs. 6(b)$6(d)$, the flow pattern is almost the inverse of that observed without vibration. The material along the inclined walls discharges first (a layer of approximately 20 particle diameters), and that in the central core discharges last. After an initial transient period, the material on the free surface is inclined at an angle of $105^{\circ}$ to the vertical. This angle remains relatively constant throughout the discharge period. The material on the free surface avalanches toward the wall and then descends along the inclined surface before discharging.

The transition from the funnel-flow to the invertedfunnel pattern occurs over a range of vibrational velocity and acceleration, affecting the inclination of the upper free surface and coinciding with an increase in the discharge rate. Figure 7 shows the variation in the inclination with acceleration for a hopper vibrating at $35 \mathrm{~Hz}$. Data are presented near the middle of the discharge, and in the last $20 \%$ of the discharge as sketched in the inset figures. The measurements were made from recorded images of the flow, and hence are accurate to within $\pm 3^{\circ}$.

For low accelerations, $\Gamma<0.5$, the flow appears to be unaffected by the vibration. As the acceleration level is increased to $\Gamma \approx 1$, the upper free surface begins to flatten, and reaches a final inclination of $105^{\circ}$ at an acceleration of approximately $\Gamma=1.1$. However, in comparing the free surface at the middle of the discharge period to that at the end, the data indicate that the fill level of the hopper also affects the transition. In the intermediate range of accelerations, 0.5 $<\Gamma<1.1$, the discharge initially follows an inverted-funnel pattern, but then changes to a funnel flow as the level of fill decreases.

The inclination free surface was also monitored at other frequencies and accelerations as shown in Fig. 8. At 25 and $30 \mathrm{~Hz}$, the maximum inclination that the surface attained was $105^{\circ}$, approximately the same as found at $35 \mathrm{~Hz}$. However, for lower frequencies, the maximum angle was not as large

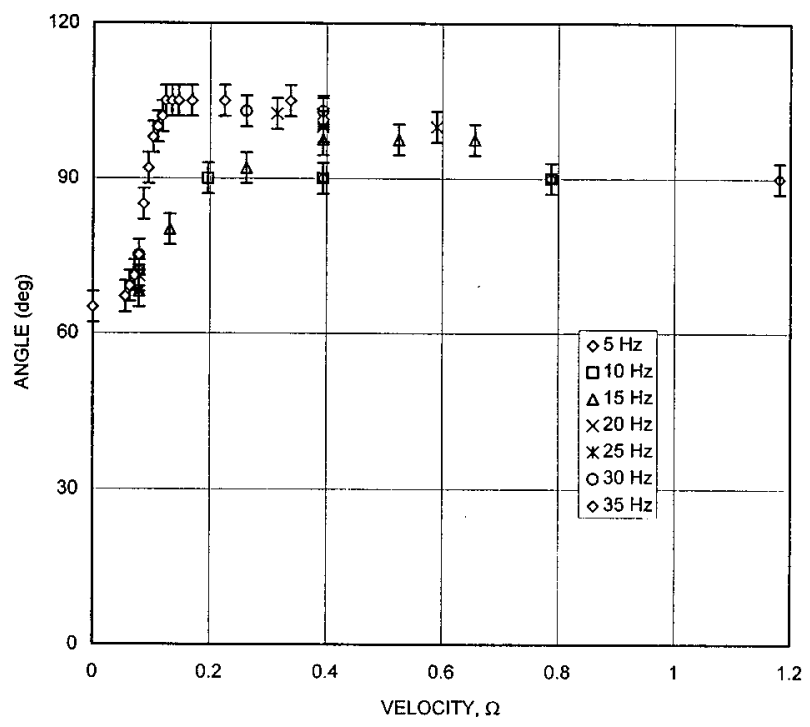

FIG. 8. Inclination of the free surface midway through the discharge for frequencies from 5 to $35 \mathrm{~Hz}$ as a function of vibration velocity, $\Omega$ $=(a \omega) /\left(g D_{h}^{\prime}\right)^{1 / 2}$.

and the upper surface became flatter. In fact for 5 and $10 \mathrm{~Hz}$, the maximum angle was $90^{\circ}$; hence, for these frequencies, the flow did not resemble the inverted-funnel pattern that was found at higher frequencies and appears to resemble that of a mass-flow hopper in which the material is discharged uniformly. Figure 8 also shows that the inclination angle reached a maximum value for vibrational velocities greater than approximately 0.2 .

In addition to the free surface, the flow near the exit of the hopper was also monitored with the high-speed camera (500 frames per s). Figure 9 shows three digitized images of the hopper at different positions within the cycle at $\Gamma=2$ and

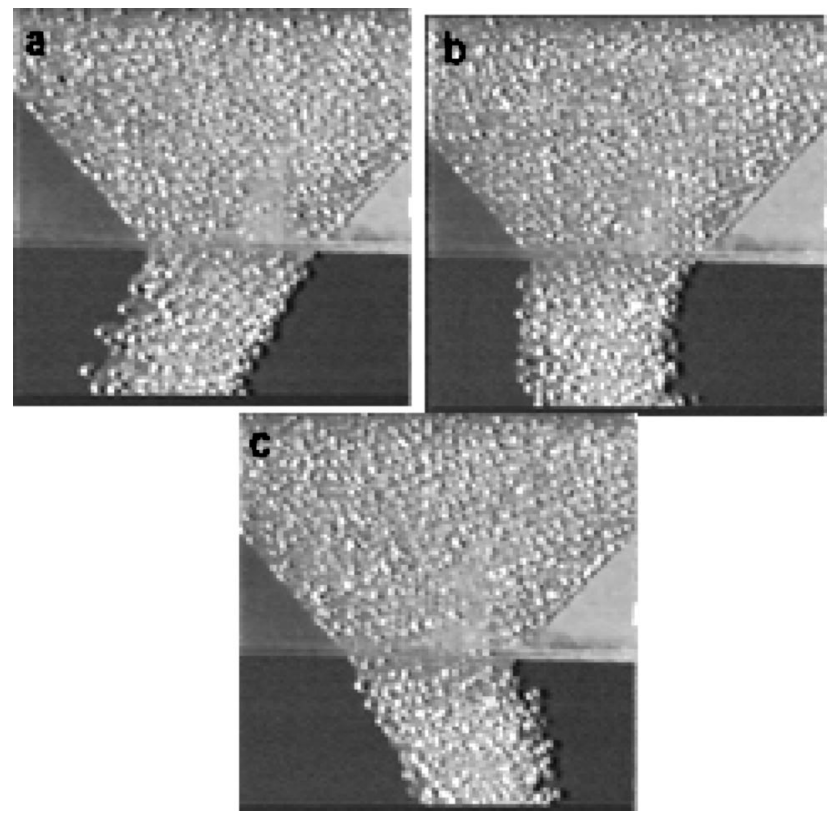

FIG. 9. Digitized images of the exit of the hopper vibrating at $20 \mathrm{~Hz}$ and $\Gamma=2.0$ at three different points during the vibration cycle; (a) $\omega t \cong 0 ;$ (b) $\omega t \cong \pi / 2 ;(\mathrm{c}) \omega t \cong \pi$. 


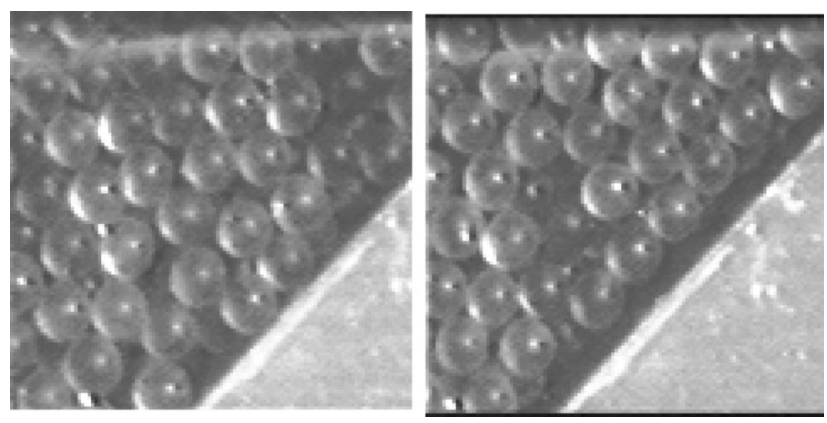

FIG. 10. Digitized images of particles near the right-hand wall of a hopper with the hopper vibrating at $20 \mathrm{~Hz}$ and $\Gamma=2.0$; the left-hand side shows the particles in contact with the wall, and the right-hand side shows a small gap between the wall and the particles.

$20 \mathrm{~Hz}$. In Fig. 9(a), the hopper is moving to the right-hand side and $\omega t \cong 0$; at this position, the inclination of the discharging material relative to the vertical reaches a maximum. All of the material exiting the hopper appears to be descending from the right-hand side of the hopper. The material in the center and left-hand side appears to remain stationary. As the hopper moves farther to the right-hand side and $\omega t$ approaches $\pi / 2$, the angle of the discharge begins to decrease, first at the left-hand side of the hopper and then at the righthand side. At $\omega t \cong \pi / 2$, the discharge is completely vertical as shown in Fig. 9(b). As the hopper moves to the left-hand side, the angle of the discharging material increases until a maximum is reached at approximately $\omega t \cong \pi$ as shown in Fig. 9(c). At this point, the material appears to be exiting primarily from the left-hand side of the hopper.

\section{MOTION OF INDIVIDUAL PARTICLES}

To understand the flow and the changes with acceleration and frequency, motions of individual particles were monitored by tracking a spot of light reflected off of the surface of a particle with a commercial frame-grabber routine. An example of the particle near the wall is shown in the digitized images of Fig. 10. The determination of the particle trajectories was limited by the number of images per cycle, which decreased for the higher frequencies. The technique did not allow any rotation of the particle to be monitored.

A sample trajectory for a particle along the inclined right wall is shown in Fig. 11 for a case $(20 \mathrm{~Hz}$ and $\Gamma=2)$ corresponding to the inverted-funnel pattern. The trajectory data begins with the particle at its rightmost position (point $A$ ). The particle then moves horizontally with the hopper. As the hopper moves to its leftmost position (point $B$ ), a gap opens between the particle and the hopper as observed in Fig. 10. The particle then follows a curved path until it falls a distance, $h$, and recontacts the hopper (point $C$ ). Subsequently, the particle follows the hopper moving first to the right-hand side (point $D$ ), and then to the left-hand side repeating the cycle.

In Fig. 12, the data are replotted in terms of the horizontal $\left(x-x_{0}\right)$ and vertical $\left(y-y_{0}\right)$ positions of the particle nondimensionalized by the amplitude of vibration, $a$, and a nondimensional time, $\omega t / 2 \pi$. Also shown are the points $A-D$ and curves corresponding to $\sin (\omega t)$ to indicate the

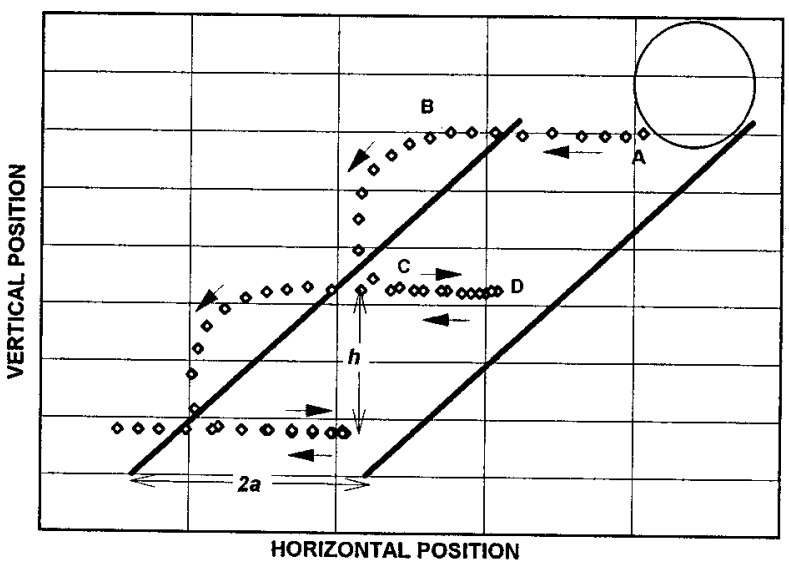

FIG. 11. Trajectory of a particle adjacent to the sidewall. The hopper is vibrating at $20 \mathrm{~Hz}$ and $\Gamma=2.0$.

position of the hopper. By comparing the position of the particle with the position of the hopper wall, it emerges that the particle leaves the surface at a time $t_{1}$ (or at a critical angle, $\left.\theta_{1}\right)$. At this moment the particle and the wall are moving to the left-hand side, but the wall is decelerating. Since the particle is not attached to the wall, it may continue to move to the left-hand side at approximately a constant velocity as long as there is available space. The hopper then reverses direction at $\omega t / 2 \pi=0.75(\theta=-\pi / 2)$, but the particle continues to move to the left-hand side. Subsequently, the particle slows down because it comes in contact with other particles; it then reverses direction to move with the other material. The particle reconnects with the sinusoidally varying wall at some later time, $t_{2}$. Examination of the vertical position of the particle during this time period shows that the particle descends only during flight. The vertical drop, $h$, appears to be approximately constant for each vi-

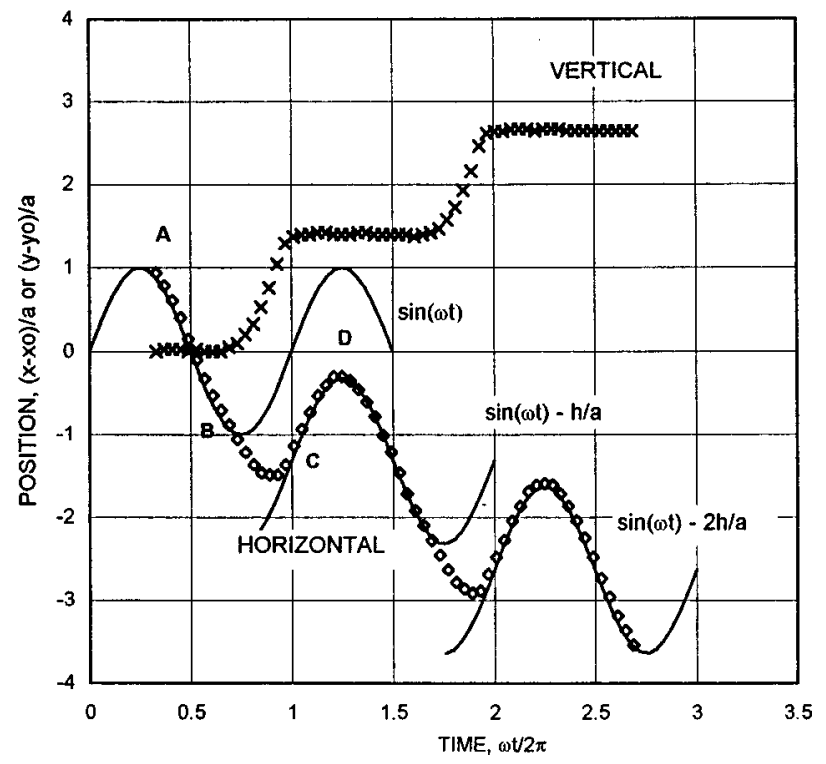

FIG. 12. Vertical, $\left(y-y_{0}\right) / a$, and horizontal, $\left(x-x_{0}\right) / a$, positions for a particle adjacent to the sidewall with the hopper vibrating at $20 \mathrm{~Hz}$ and $\Gamma$ $=2.0$. 


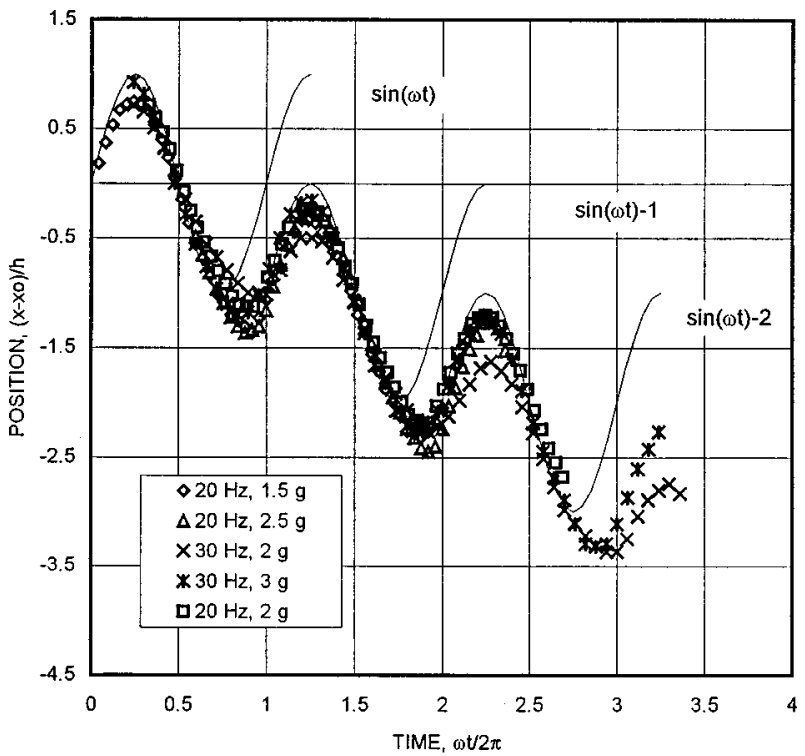

FIG. 13. Horizontal, $\left(x-x_{0}\right) / h$, positions for a particle adjacent to the sidewall with the hopper vibrating at conditions that correspond with an inverted-funnel pattern.

bration cycle; the value of $h$ is used to define the offset of the other sinusoidal curves shown in Fig. 12. Hence, after the first flight, the particle follows a trajectory that is offset from the original $a \sin (\omega t)$ by an amount $(h / \tan \alpha)$ (in the current experiments the offset is $h$ since $\tan \alpha=1$ ). Afterwards the particle reconnects with the sinusoidal curve given by $\sin (\omega t)-h / a$, for times $\left(\omega t_{2} / 2 \pi\right)<(\omega t / 2 \pi)<1+\left(\omega t_{1} / 2 \pi\right)$. The flight of the particle is repeated every cycle.

Figures 13 ( $x$ positions) and 14 ( $y$ positions) show similar single-particle information for different accelerations be-

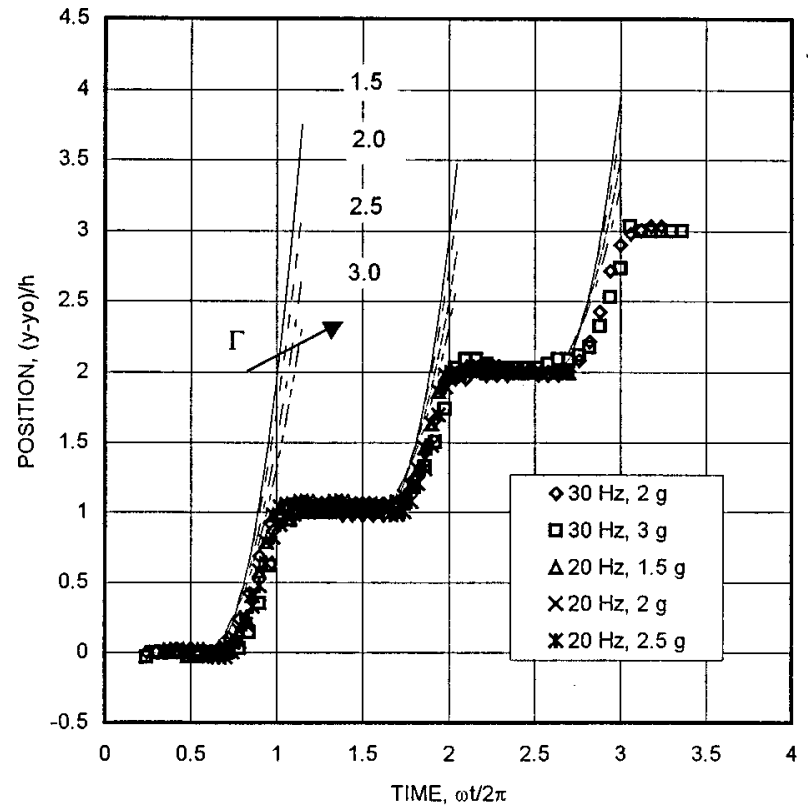

FIG. 14. Vertical $(y / h)$ positions for a particle adjacent to the sidewall with the hopper vibrating at conditions that correspond to an inverted-funnel pattern. Lines show the trajectory for a particle falling under gravity with the initial starting time corresponding to $\theta_{1}=a \sin (-1 / \Gamma)$.

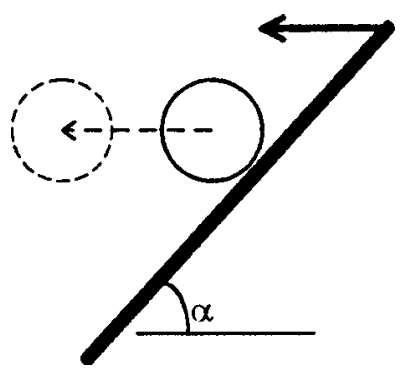

FIG. 15. Particle leaving the inclined surface as the hopper decelerates while moving to the left-hand side.

tween $1.5<\Gamma<3$ and frequencies of 20 and $30 \mathrm{~Hz}$. The positions, however, are nondimensionalized by the vertical jump, $h$, for that particular experiment. As shown in Fig. 13, the particle follows the sinusoidal path except during the flight of the particle. In Fig. 14, the vertical displacements of the particles follow a repeatable pattern, a parabolic trajectory resulting from the gravitational force acting on the particle during the free fall in flight. Hence, the vertical position of the particle can be determined from

$$
y / a=g t^{\prime 2} /(2 a)=\left(\omega t^{\prime}\right)^{2} /(2 \Gamma),
$$

where $t^{\prime}$ represents the time of flight of the particle, $t^{\prime}=t$ $-t_{1}$.

An estimate for the time $t_{1}$ can be obtained from a simple analysis of a single particle moving on an inclined plate, such as shown in Fig. 15. In the frame accelerating with the particle, the acceleration normal to the plate is $g \cos \alpha+a \omega^{2} \sin \theta_{1} \sin \alpha$, and therefore contact with the plate will be lost when

$$
g \cos \alpha=-a \omega^{2} \sin \theta_{1} \sin \alpha
$$

and rearranging

$$
\Gamma \sin \theta_{1} \tan \alpha=-1 .
$$

For $\tan \alpha=1$, then $\theta_{1}=\arcsin (-1 / \Gamma)$ and $\omega t_{1}=-\theta_{1}+\pi$.

In Fig. 14, the lines for the parabolic trajectory are given in terms of $y / h=g t^{\prime 2} /(2 h)=(a / h)\left(\omega t^{\prime}\right)^{2} /(2 \Gamma)$, with the origin of the trajectory starting from the time $t_{1}$ given by Eq. (5). The predictions for $t_{1}$ indicate an earlier lift-off point than the experimental data. However, the difference is small; it may be a result of the dilation of the bed since the model assumes that there is space in which the particle can move when it leaves the surface. A second possibility is that the particle does not leave the surface until the plate reverses directions. Then lift-off occurs when $\omega t_{1} / 2 \pi=0.75$. Figure 16 is similar to Fig. 14, but the lift-off time used in determining the parabolic trajectories is given by the second criterion. It appears that the lift-off occurs between these two limiting criteria, $-\arcsin (-1 / \Gamma)+\pi<\omega t_{1}<3 \pi / 2$, with the exact time of departure of the particle determined by the movement of other particles within the hopper.

When the particle is in flight, the flight time determines the vertical distance $h$ that the particle will move during each period of oscillation. Values of $h / a$ for different accelerations and frequencies are given in Fig. 17. The results suggest that there is a maximum value of $h / a$ for a particular 


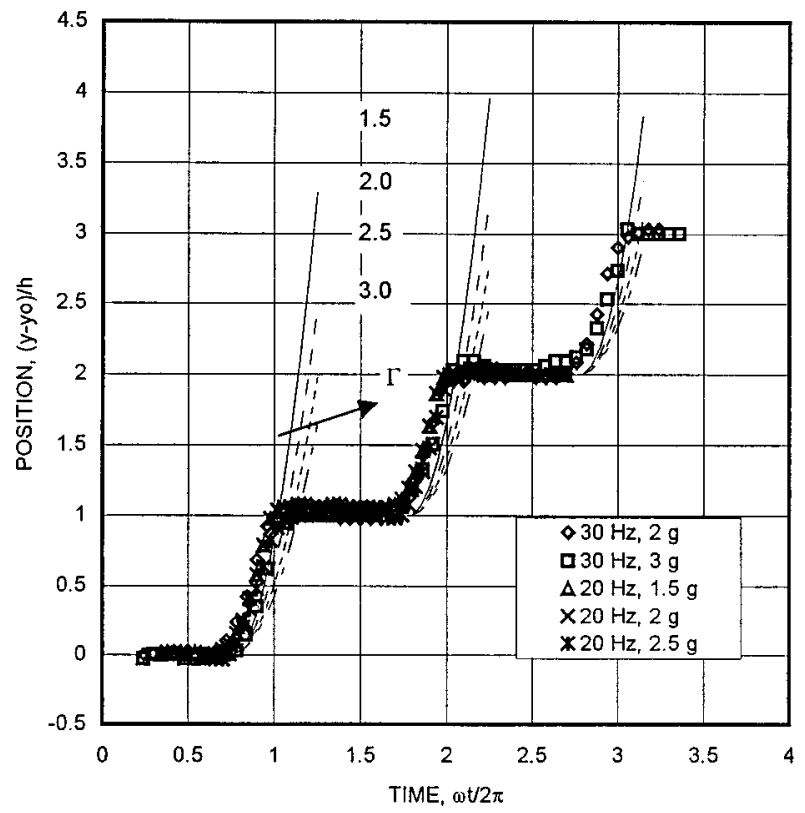

FIG. 16. Vertical $(y / h)$ positions for a particle adjacent to the sidewall with the hopper vibrating at conditions that correspond to an inverted-funnel pattern. Lines show the trajectory for a particle falling under gravity with the initial starting time corresponding to $\theta_{1}=-\pi / 2$.

frequency. The error bars reflect the resolution of the images of the particles and the variability in $h$ from particle to particle.

An estimate for the time of flight $\tau$ and the distance $h$ can be found from a model of the particle on an inclined surface as shown in Fig. 18. The particle will lose contact with the surface as the surface is moved in the $x$ direction. However, if the surface decelerates (as in the case of a sinusoidal vibration), the particle and the surface will reconnect. Beginning with the plate at the maximum displacement from

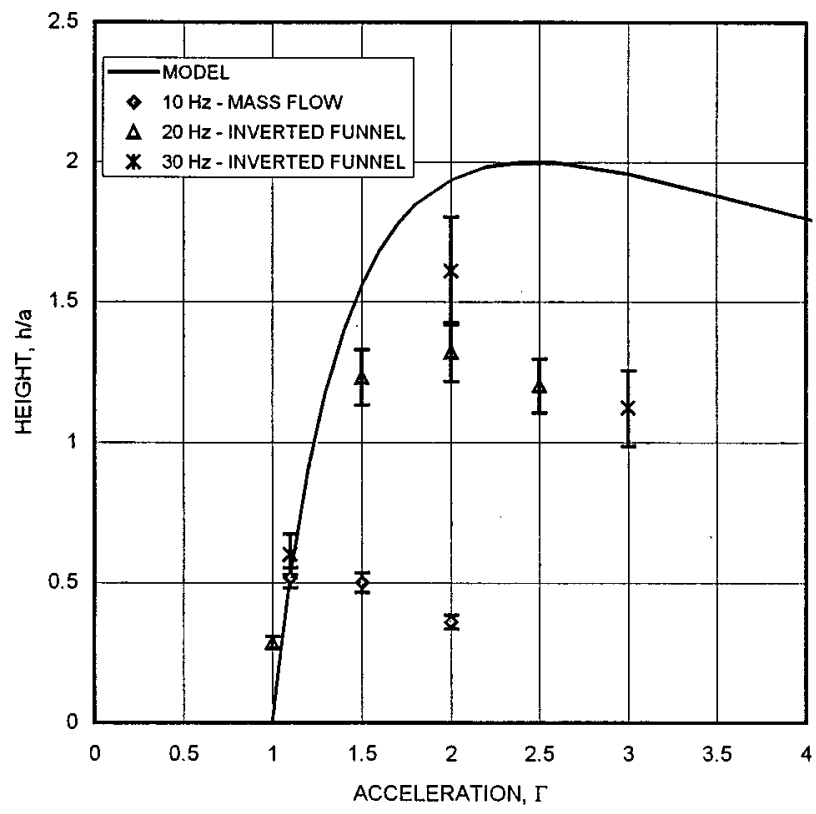

FIG. 17. Vertical distance, $h / a$, moved during each cycle of vibration by a particle adjacent to the sidewalls. The solid line corresponds to a model from a single particle on vibrating plate, Eq. (8).

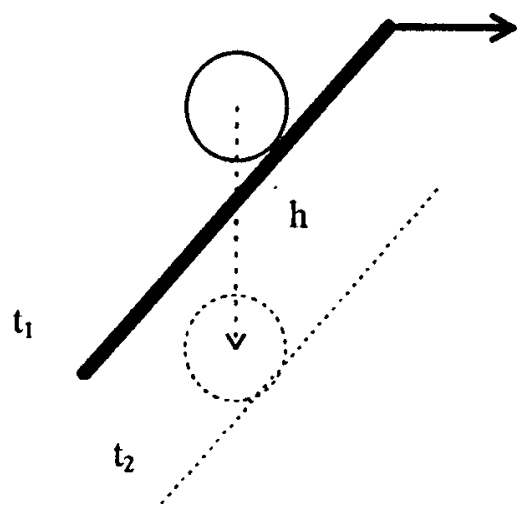

FIG. 18. Vertical displacement of a particle due to horizontal motion and subsequent deceleration of the inclined plate.

the centerline, the plate moves a horizontal distance, $a[1$ $-\cos (\omega \tau)]$, in time $\tau$. After this period $\tau$, a vertical gap of distance $a[1-\cos (\omega \tau)] \tan \alpha$ opens for the particle to fall. If the particle has no initial vertical velocity, the particle and the surface will reconnect when

$$
g \tau^{2} / 2=a[1-\cos (\omega \tau)] \tan \alpha,
$$

which can be rewritten as

$$
(\omega \tau)^{2} /[1-\cos (\omega \tau)]=2 \Gamma \tan \alpha
$$

and solved for $\tau$. Figure 19 shows the roots of Eq. (7) in terms of $\omega \tau$ as a function of $\Gamma \tan \alpha$. There are no solutions to the equation for $\Gamma \tan \alpha<1$ and under these conditions, the particle does not separate from the surface. For $\Gamma \tan \alpha$ $>1, \omega \tau$ increases with acceleration level. The distance that the particle falls is then

$$
\frac{h}{a}=[1-\cos (\omega \tau)] \tan \alpha,
$$

which is indicated in Fig. 17 for $\tan \alpha=1$. The estimate overpredicts the experimental data for all frequencies; the model,

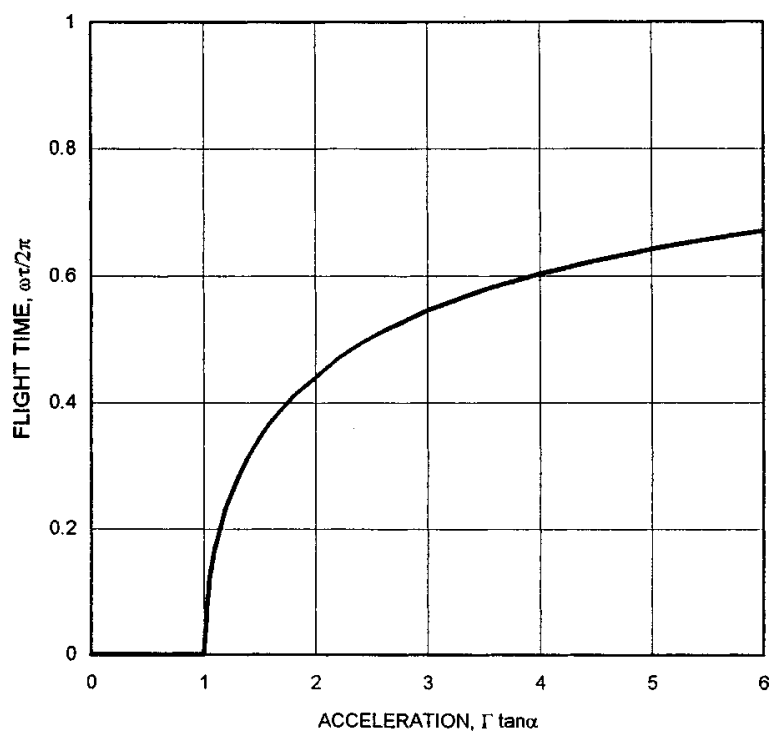

FIG. 19. Nondimensional flight time predicted as a function of acceleration $(\Gamma \tan \alpha)$ from a model of a single particle on a vibrating plate. 


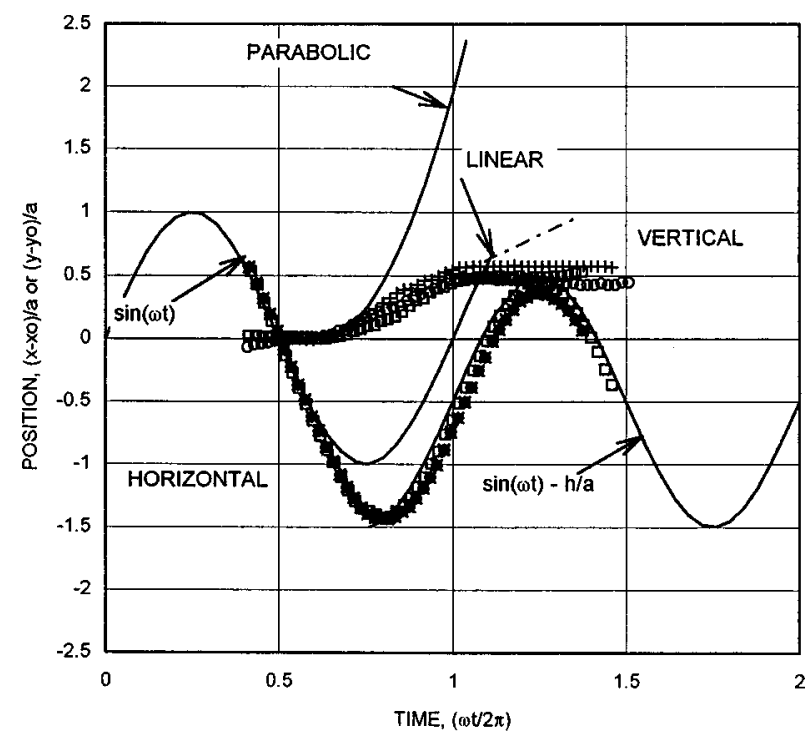

FIG. 20. Horizontal $(x / a)$ and vertical $(y / a)$ positions for a particle adjacent to the sidewall with the hopper vibrating at $10 \mathrm{~Hz}$ and $\Gamma=1.5$. The lines show a trajectory if the vertical position increased linearly with time and the initial starting time corresponded to $\theta_{1}=\arcsin (-1 / \Gamma)$.

however, does indicate a peak with acceleration. From the experimental trajectories, it was observed that the particle separates from the surface prior to the hopper changing directions. Hence, the particle and the hopper initially move in the same direction, which would tend to decrease the overall flight time of the particle as compared with the prediction from the model.

In addition to the flight time, the model can be used to predict an average downward velocity of the particles by dividing the vertical distance, $h$, by the period of oscillation, $(2 \pi / \omega)$. Hence, an average downward speed, $V_{a}$, scaled by the amplitude of the vibrational velocity is

$$
\frac{V_{a}}{a \omega}=\frac{1}{2 \pi} \frac{h}{a}=\frac{1-\cos \omega \tau}{2 \pi} \tan \alpha,
$$

where $\tau$ is a function of $\Gamma$ as given in Fig. 19. The expression for $V_{a} /(a \omega)$ shows a relatively weak dependence on acceleration for $\Gamma>1.5$. Hence, the downward velocity depends primarily on the vibration velocity $(a \omega)$, which appears to be consistent with the discharge measurements as a function of vibrational velocity given in Fig. 4.

For lower frequency, the flow differs from the invertedfunnel flows found at higher frequencies. In Fig. 20, data are plotted for the $x$ and $y$ positions over a single vibration cycle for a frequency of $10 \mathrm{~Hz}$ and $\Gamma=1.5$. Data from three different particles are shown. The vertical positions of the particle do not follow a parabolic path, but instead are more closely represented by a linear variation as shown by the curve in Fig. 20. The slope of the line is chosen to best fit the data, and has a value of 1.3. From the high-speed images, it appears that the particle never separates from the hopper, but instead rolls or slides along the surface of the hopper for a period of time. During the remainder of the vibration cycle, the particle moves with the hopper. The data for the ratio of fall height to amplitude of vibration is also plotted in Fig. 17.
The values at $10 \mathrm{~Hz}$ are smaller than at 20 or $30 \mathrm{~Hz}$, and deviate considerably from the model.

\section{SUMMARY AND CONCLUSION}

The present work shows that horizontal vibration can increase the discharge rate from a hopper and eliminate the first-in last-out problem that is encountered in funnel-flow designs. For $\Gamma>1$ and higher frequencies (above $20 \mathrm{~Hz}$ ), the flow exhibits an inverted-funnel pattern as reflected in the surface angle of the free surface and by visualization of the flow. For inverted-funnel flows, the particles near the wall are in free flight over part of the vibration cycle. This free flight occurs just prior to the point that the hopper reverses direction. The distance that the particle falls during the flight can be estimated from a simple model of a sphere on a moving inclined plate. The discharge from the hopper occurs first from one side of the hopper, and then from the other, with little relative movement in the center.

For lower frequencies $(5$ and $10 \mathrm{~Hz}$ ), the free surface of the material remains relatively flat, indicating that the material is discharged uniformly over one oscillation cycle. The particles along the sidewalls do not fall under gravity but roll or slide along the inclined walls during the period of time when the hopper is decelerating and as the hopper reverses directions. For small vibrational velocities, $\Omega<0.2$, observations of the free surface and the discharge rates indicate that vibration has little effect on the flow; hence the flow remains as a funnel flow. The depth of the material in the hopper also affects the type of discharge.

In the current experiments, the flows involved material of approximately equal size spheres. For stationary hoppers that discharge mixtures of materials, segregation of the material according to size or composition is a common phenomena. An interesting topic for future studies would involve the effect of horizontal vibration on discharging mixtures of materials. The horizontal vibration could be used to minimize stagnant regions, which could reduce segregation of the mixture.

${ }^{1}$ P. A. Shamlou, Handling of Bulk Solids, Theory and Practice, 1st ed. (Butterworths, London, 1988).

${ }^{2}$ R. M. Nedderman, Statics and Kinematics of Granular Materials, 1 st ed. (Cambridge University Press, Cambridge, 1992).

${ }^{3}$ W. A. Beverloo, H. A Leniger, and J. Van de Velde, "The flow of granular solids through orifices," Chem. Eng. Sci. 15, 260 (1961).

${ }^{4}$ T. V. Nguyen, C. E. Brennen, and R. H. Sabersky, "Funnel flows in hoppers," J. Appl. Mech. 47, 729 (1980).

${ }^{5} \mathrm{~K}$. Wieghardt, "Experiments in granular flow," Annu. Rev. Fluid Mech. 89-114 (1975).

${ }^{6}$ C. R. Wassgren, "Vibration of granular materials," doctoral thesis, California Institute of Technology, 1996.

${ }^{7}$ A. Suzuki, H. Takahashi, and T. Tanaka, "Behavior of a particle bed in the field of vibration. II. Flow of particles through slits in the bottom of a vibrating vessel," Powder Technol. 2, 72 (1968).

${ }^{8} \mathrm{P}$. Evesque and W. Meftah, "Mean flow of a vertically vibrated hourglass,” Int. J. Mod. Phys. B 7, 1799 (1993).

${ }^{9}$ G. H. Ristow, X. Strabburger, and X. Rehberg, "Phase diagram and scaling of granular materials under horizontal vibrations," Phys. Rev. Lett. 79, 833 (1997).

${ }^{10}$ K. Liffman, G. Metcalfe, and P. Cleary, "Granular convection and transport due to horizontal shaking," Phys. Rev. Lett. 79, 4574 (1997). 